() И.В. Друк ${ }^{1 *}$, Д.И. Снарская ${ }^{1}$, О.И. Горощеня²

'Омский государственный медицинский университет, Омск

${ }^{2}$ Городская поликлиника №13, Омск

ОБОСНОВАНИЕ. Общая численность пациентов с сахарным диабетом 2 типа (СД2) в РФ на 01.01.2019 составляет 4,24 млн. Основную долю больных СД2 составляют люди пожилого возраста и старше, формирующие группы с высокой коморбидностью и риском тяжелой гипогликемии, в том числе связанной с избыточностью сахароснижающей терапии. Зарубежные исследования свидетельствуют, что значительная доля пожилых людей, страдающих диабетом, потенциально подвергаются избыточной сахароснижающей терапии.

ЦЕЛЬ. Изучить частоту избыточного снижения уровня гликированного гемоглобина (НbА 1 ) в группе пациентов с СД2 по данным выборки из регионального регистра СД.

МЕтоДЫ. Проведен контент-анализ регионального регистра сахарного диабета по данным на 31.12.2019. На основании данных регистра рассчитывался индивидуальный целевой уровень $\mathrm{HbA}_{1 c^{\prime}}$ оценивалось соответствие достигнутого уровня $\mathrm{HbA}_{1 \mathrm{c}}$ целевому диапазону.

РЕзУЛЬтАТЫ. В анализ включены данные 1202 пациентов с СД2, что составило 2,35\% от общей численности пациентов с СД2 в регионе ( $n=51$ 163). Средний возраст включенных лиц - 66 лет (LQ 60,0; UQ 72), из них мужчин - 360 (29,95\%). Длительность СД2 составила 8,0 года. Уровень НbА составляли 75,21\% (n=904). $\mathrm{HbA}_{1 c}$ находился выше целевого уровня в 43,34\% случаев (n=521). Уровень $\mathrm{HbA},<6,5 \%$ отмечался в четверти случаев (24,62\%), в том числе $\mathrm{HbA}_{1 c}<6,0 \%$ был зарегистрирован в 97 случаях, что составило треть всех случаев жесткого контроля гликемии. При этом большинство наблюдений НbА 1 <6,5\% приходилось на пациентов 60 лет и старше (79,73\%). Среди пациентов молодого и среднего возраста с НbA $<6,5 \%$ в 8,33\% случаев имелись факторы риска тяжелой гипогликемии при наличии терапии препаратами сульфонилмочевины (СМ) и/или инсулином. В старшей возрастной группе (в сравнении с пациентами молодого и среднего возраста) значимо чаще выявлялся $\mathrm{HbA}_{1 c}<7 \%(\mathrm{p}<0,05)$, имелась тенденция к большей частоте $\mathrm{HbA}_{1 c}<6,5 \%(\mathrm{p}=0,067)$. Среди пациентов пожилого и старческого возраста, имевших $\mathrm{HbA}_{1 c}<6,5 \%$, в 41,53\% наблюдений имелись факторы риска тяжелой гипогликемии, в четверти случаев в терапии СД2 применялись СМ и/или инсулин (24,58\%), практически каждый пятый пациент (19,07\%), имевший факторы риска тяжелой гипогликемии, получал СМ и/или инсулин.

ЗАКЛЮчЕНИЕ. По нашим данным, как минимум четверть пациентов старшей возрастной группы (24,58\%) нуждаются в деинтенсификации терапии. Можно полагать, что в этой группе пациентов риски, связанные с лечением, могут преобладать над преимуществами жесткого контроля гликемии, и эти пациенты нуждаются в плановой деинтенсификации сахароснижающей терапии. Принимая во внимание позицию некоторых экспертных сообществ по определению целевого диапазона $\mathrm{HbA}_{1 с}$ в старшей возрастной группе, потребность в деинтенсификации сахароснижающей терапии может быть еще более высокой. Требуются дальнейшие исследования с целью разработки полноценной отечественной концепции деинтенсификации сахароснижающей терапии.

КЛЮЧЕВЫЕ СЛОВА: сахарный диабет 2 типа; избыточность терапии; клиническая практика

\title{
POTENTIAL OVERTREATMENT OF TYPE 2 DIABETES THERAPY IN REAL CLINICAL PRACTICE: OMSK OBLAST REGISTER DATA
}

\author{
(c) Inna V. Druk ${ }^{1 *}$, Diana I. Snarskaya' ${ }^{1}$ Oksana I. Goroshchenya ${ }^{2}$
}

'Omsk State Medical University, Russian Federation, Omsk, Russia

${ }^{2}$ City Polyclinic №13, Omsk, Russia

BACKGRAUND: The total number of patients with type 2 diabetes mellitus (T2DM) in the Russian Federation as of 01.01 .2019 is 4.24 million. The majority of patients with T2DM are elderly people and older, forming groups with high comorbidity and the risk of severe hypoglycemia, including those associated with excess hypoglycemic therapy. Foreign studies indicate that a significant proportion of older adults with diabetes are potentially exposed to excessive sugar-lowering therapy.

AIMS: to study the frequency of excessive decrease in $\mathrm{HbA}_{1 \mathrm{c}}$ in a group of patients with T2DM according to a sample from the regional diabetes register. 
MATERIALS AND METHODS: A content analysis of the regional register of diabetes mellitus was carried out as of December 31, 2019. Based on the register data, an individual target level of $\mathrm{HbA}_{1 \mathrm{c}}$ was calculated and the compliance of the achieved $\mathrm{HbA}_{1 c}$ level with the target level was assessed.

RESULTS: The analysis included data from 1202 patients with T2DM, which amounted to $2.35 \%$ of the total number of patients with T2DM in the region $(n=51,163)$. The age of the included individuals was 66 years (LQ 60.0; UQ 72), 360 men (29.95\%). The duration of T2DM 8.0 years. The $\mathrm{HbA}_{1 c}$ level in the general group was $7.1 \%$. Persons over 60 years of age accounted for $75.21 \%(n=904)$. When analyzing $\mathrm{HbA}_{1 \mathrm{c}}$ in the age groups, there were no statistically significant differences $(p>0.05) . H_{b A}$ was above the target level in $43.34 \%$ of cases $(n=521)$. The level of $\mathrm{HbA}_{1 c}<6.5 \%$ was noted in a quarter of cases (24.62\%), including $\mathrm{HbA}_{1 \mathrm{c}}<6.0 \%$ was recorded in 97 cases, which accounted for a third of all cases of tight glycemic control. At the same time, the majority of observations of $\mathrm{HbA}_{1 \mathrm{c}}<6.5 \%$ were in patients 60 years and older (79.73\%). Among young and middle-aged patients with $\mathrm{HbA}_{1 \mathrm{c}}<6.5 \%, 8.33 \%$ of cases had risk factors for severe hypoglycemia in the presence of SU and / or insulin therapy. In the older age group (in comparison with young and middle-aged patients), $\mathrm{HbA} \mathrm{Ac}_{1 \mathrm{c}}<7 \%$ $(p<0.05)$ was significantly more often detected, there was a tendency for a higher frequency of $\mathrm{HbA}_{1 c}<6.5 \%(p=0.067)$. Among elderly and senile patients with $\mathrm{HbA}_{1 c}<6.5 \%$, in $41.53 \%$ of cases there were risk factors for severe hypoglycemia, in a quarter of cases in T2DM therapy SU and / or insulin were used (24.58\%), almost every fifth patient (19.07\%), risk factors for severe hypoglycemia, received SU and / or insulin.

CONCLUSION: According to our data, at least a quarter of patients in the older age group (24.58\%) had overtreatment and need de-intensification of therapy. Perhaps that in this group of patients, the risks associated with treatment may outweigh the benefits of tight glycemic control, and these patients require planned de-intensification of glucose-lowering therapy. Taking into account the position of some expert communities on determining the target range of $\mathrm{HbA}_{1 c}$ in the older age group, the need for de-intensification of antihyperglycaemic therapy may be even higher. Further research is required in order to develop a full-fledged domestic concept of de-intensification of hypoglycemic therapy.

KEYWORDS: type 2 diabetes; overtreatment; clinical practice

По данным онлайн-регистра, общая численность пациентов с сахарным диабетом (СД) в РФ на 01.01.2019 составляет 4,58 млн (3,12\%), в том числе СД 2 типа (СД2) 4,24 млн. С 2000 г. численность пациентов с СД в РФ выросла в 2,2 раза [1]. Согласно актуальным клиническим рекомендациям по терапии СД, принята концепция индивидуального целевого уровня $\mathrm{HbA}_{1 с}$ [2]. Основную долю больных СД2 составляют люди пожилого возраста и старше, формирующие группы с высокой коморбидностью и риском тяжелой гипогликемии, в том числе связанной с избыточностью сахароснижающей терапии. По российским данным, пациенты с СД2 в возрасте 60 лет и старше составляют большинство как в группе женщин (77,1\%), так и в группе мужчин $(63,4 \%)$ [3].

Зарубежные исследования свидетельствуют, что значительная доля пожилых людей, страдающих диабетом, потенциально подвергаются избыточной сахароснижающей терапии [4-6].

\section{ЦЕЛЬ}

Изучить частоту избыточного снижения $\mathrm{HbA}_{1 с}$ в группе пациентов с СД2 по данным выборки из регионального регистра СД.

\section{МЕТОДЫ}

Работа выполнена на базе БУзОО ГП №13. Выбор лечебно-профилактического учреждения обосновывался наибольшей численностью пациентов с СД, наблюдающихся в учреждении, высоким качеством ведения регистра СД, участием в заполнении регистра врачей-эндокринологов (в 2 амбулаторных эндокринологических отделениях работают 10 врачей специалистов-эндокринологов). Проведен контент-анализ регионального регистра СД на 31.12.2019 после одномоментной выгрузки данных по признаку «БУЗОО ГП №13». Критерии включения данных в анализ: обезличенные (отсутствие Ф.И.О., СНИЛС) данные пациентов с СД2 старше 18 лет, включающие дату рождения, пол, возраст, год установления диагноза, схему терапии (классы, препараты), осложнения, сопутствующие заболевания, уровень гликированного гемоглобина $\left(\mathrm{HbA}_{1 c}\right)$. Определение $\mathrm{HbA}_{1 c}$ выполнялось методом жидкостной ионообменной хроматографии высокого давления на анализаторах гликозилированного гемоглобина D-10 (Bio-Rad Laboratories, США) в условиях БУЗОО «Клинический диагностический центр». Критерии исключения: сахарный диабет 1 типа, неполнота сведений, ошибки ввода данных. В соответствии с критериями включения/исключения из общей выборки, содержащей сведения о 1451 пациенте, в анализ были включены данные 1202 пациентов (82,84\%). Проверка нормальности распределения производилась с использованием метода Шапиро-Уилка. При законе распределения, отличном от нормального, данные записывались как медиана, верхний (UQ) и нижний (LQ) квартили. Проводился анализ таблиц сопряженности для двух независимых групп с применением критерия $\mathrm{X}^{2}$ (с поправкой Йейтса при малом количестве наблюдений), при сравнении рядов непрерывных количественных данных - корреляционный анализ (критерий Спирмена). Для поиска статистически значимых связей между количественными непрерывными и дискретными порядковыми данными рассчитывалось кор-

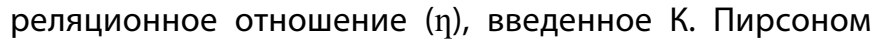
(показатель нелинейной связи по отношению одного параметра к другому). Для сравнения независимых выборок по количественному признаку использовались критерии Краскела-Уоллиса (Н) и Манна-Уитни (U). Критическое значение уровня значимости (p) принималось 


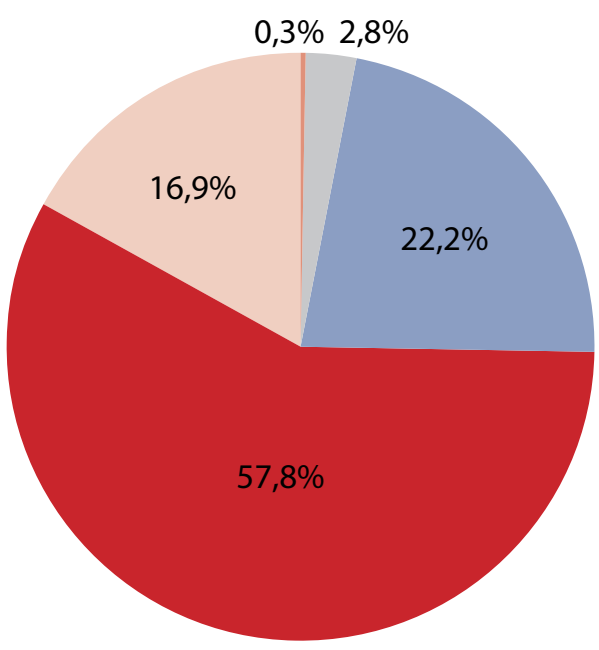

Молодой возраст (18-14 лет)

Средний возраст (45-59 лет)

Пожилой возраст (60-74 года)

Старческий возраст (75-89 лет)

Долгожители (90 лет и старше)

Рисунок 1. Характеристика пациентов по возрастным группам.

равным 5\%. Статистический анализ проводился с использованием программных пакетов анализа Microsoft Excel, Statistica 13.0 (StatSoft Inc., США, trial-версия).

\section{РЕЗУЛЬТАТЫ}

В анализ включены данные 1202 пациентов с СД2, что составило 2,35\% от общей численности пациентов с СД2 в регионе ( $\mathrm{n}=51$ 163). Средний возраст включенных лиц составил 66 лет (LQ 60,0; UQ 72), из них мужчин - 360 (29,95\%). Средняя длительность СД2 составила 8,0 года (LQ 3,0; UQ 13,0). Возрастная характеристика группы: молодой возраст (до 45 лет) - 33 пациента, средний возраст (45-60 лет) - 265 пациентов, пожилой возраст (60-75 лет) - 697 пациентов, старческий возраст (75-90 лет) - 203 пациента, возраст долгожителя (старше 90 лет) - 4 пациента. Из 1202 пациентов, которые были включены в анализ, 75,21\% (n=904) составляли лица старше 60 лет, 25\% (n=298) относились к возрастной группе от 18 до 59 лет (рис. 1).

По данным регистра, в рассматриваемой группе пациентов осложнения диабета были зарегистрированы в 64,22\% случаев (n=772). Среди зарегистрированных осложнений на первом месте была диабетическая нейропатия ( $\mathrm{n}=452,37,60 \%)$, на втором месте - макроангиопатия $(\mathrm{n}=309 ; 25,71 \%)$, на третьем месте - диабетическая нефропатия $(\mathrm{n}=229 ; 19,05)$, диабетическая ретинопатия (n=183; 15,22\%). Диабетическая катаракта регистрировалась в 32,20\% (n=387) случаев, синдром диабетической стопы - у 42 пациентов (3,49\%), хроническая сердечная недостаточность (ХCH) зафиксирована в 23,88\% случаев $(n=287)$. Острые сосудистые катастрофы (цереброваскулярные заболевания (острое нарушение мозгового кровообращения, инсульт) и/или инфаркт миокарда) были зарегистрированы в 10,48\% наблюдений $(n=126)$. Ассоциированная артериальная гипертензия имела место у 885 пациентов (73,63\%).

В целом с увеличением возраста пациентов снижалась доля лиц с отсутствием зарегистрированных осложнений СД2 (табл. 1).

Закономерно была зарегистрирована сильная положительная корреляция распространенности осложнений СД с возрастом ( $r=0,68 ; p<0,05)$. Длительность СД2 различалась в возрастных группах (критерий КраскелаУоллиса; $\mathrm{p}<0,001$ ): длительность заболевания возрастала к каждой последующей возрастной группе (для любых парных сравнений $р<0,001)$. Среди пациентов старшей возрастной группы (пожилой и старческий возраст) значимо реже регистрировалось отсутствие осложнений СД, значимо чаще - 2 и более осложнений, выявляемых в целом в 35,39\% случаев (табл. 2).

Таблица 1. Характеристика длительности и осложнений сахарного диабета 2 типа в различных возрастных группах пациентов

\begin{tabular}{ccccccc}
\hline \multirow{2}{*}{$\begin{array}{c}\text { Возрастная } \\
\text { группа, лет }\end{array}$} & $\begin{array}{c}\text { Количество } \\
\text { пациентов/ } \\
\text { мужчин, абс. }\end{array}$ & $\begin{array}{c}\text { Длитель- } \\
\text { ность СД2, лет }\end{array}$ & \multicolumn{3}{c}{ Осложнения СД, абс./\% } \\
\cline { 5 - 8 } & $33 / 17$ & $2,0(1,0 ; 3,5)$ & $28 / 84,85$ & $4 / 12,12$ & $1 / 3,03$ & $\mathbf{2}$ \\
\hline 45 & $265 / 97$ & $5,0(2,0 ; 10,0)$ & $151 / 56,98$ & $57 / 21,51$ & $38 / 14,34$ & $19 / 7,17$ \\
$45-59$ & $697 / 199$ & $8,0(4,0 ; 13,0)$ & $291 / 41,75$ & $193 / 27,69$ & $130 / 18,65$ & $83 / 11,91$ \\
$60-74$ & $207 / 47$ & $12,0(6,0 ; 18,0)$ & $53 / 26,60$ & $47 / 22,71$ & $47 / 22,71$ & $60 / 28,99$ \\
\hline $75-90$ & & &
\end{tabular}

Таблица 2. Частота регистрации осложнений сахарного диабета 2 типа в основных возрастных группах

\begin{tabular}{lccc}
\hline \multirow{2}{*}{ Осложнения СД } & \multicolumn{2}{c}{ Возраст пациентов } & \multirow{2}{*}{$\mathbf{X}^{\mathbf{2}} ; \mathbf{p}$} \\
\cline { 2 - 4 } & $\begin{array}{c}\text { молодой и средний } \\
\text { (18-59 лет), N=298 }\end{array}$ & $\begin{array}{c}\text { пожилой и старческий } \\
\text { (60 лет и старше), N=904 }\end{array}$ & 43,$298 ; 0,001$ \\
\hline Нет, абс./\% & $179 / 60,07$ & $344 / 38,05$ & 4,$095 ; 0,043$ \\
1, абс./\% & $61 / 20,47$ & $240 / 26,55$ & 5,$976 ; 0,015$ \\
2, абс./\% & $39 / 13,09$ & $177 / 19,58$ & 16,$337 ; 0,001$ \\
Более 2, абс./\% & $19 / 6,38$ & $143 / 15,92$ & \\
\hline
\end{tabular}


Заболевания/состояния, определяющие риск неблагоприятных последствий гипогликемии (заболевания атеросклеротического генеза, синдром диабетической стопы, хроническая болезнь почек 3-5 стадий, большая продолжительность СД), в общей группе регистрировались в 38,27\% случаев ( $n=460)$, чаще - среди лиц пожилого/старческого возраста в сравнении с пациентами молодого/среднего возраста (44,35\% и 19,79\% соответственно; $\left.X^{2}=56,189 ; p=0,001\right)$.

Терапия СД2 была представлена только немедикаментозной в 5,57\% (n=67) случаев, остальные пациенты получали сахароснижающую терапию: инсулинотерапию - 26,87\% (n=323), неинсулиновые сахароснижающие препараты - 67,55\% (n=812) случаев. Следует отметить, что в общей группе 606 пациентов $(50,42 \%)$ получали препараты, характеризующиеся повышенным риском гипогликемии (препараты сульфонилмочевины (ПСМ) и/или инсулин). Среди ПСМ наиболее часто применялся гликлазид MB (n=303; 96,20\%), значительно реже - глибенкламид $(n=11 ; 3,20 \%)$, глимепирид ( $n=2 ; 0,58 \%)$. Характеристика сахароснижающей терапии в возрастных группах представлена в табл. 3.

Уровень $\mathrm{HbA}_{1 с}$ составил 7,1\% (LQ 6,5; UQ 8,5). При этом у половины пациентов с СД2 (n=602; 50,08\%) регистрировался уровень $\mathrm{HbA}_{1 с}$ в 2 крайних диапазонах значений, в соотношении 1:1 ( $<6,5 \%$ и >8,5\%) (рис. 2).

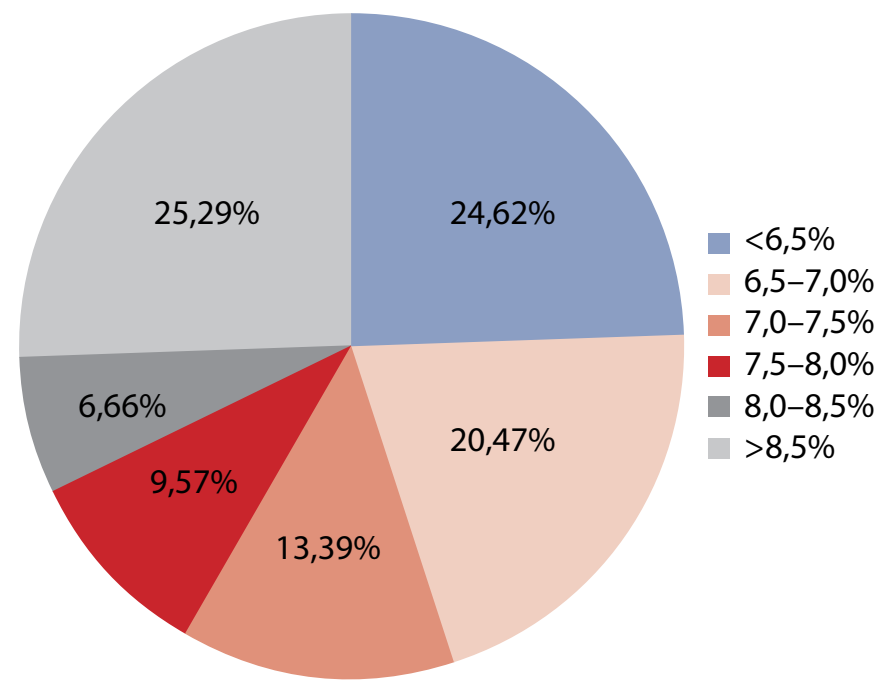

Рисунок 2. Характеристика достигнутого уровня гликированного гемоглобина, 2019 г. (n=1202).

$\mathrm{HbA}_{1 \mathrm{c}}<7 \%$ регистрировался в 45,09\% случаев, 7-7,9\% у 22,96\% пациентов. Имея сопоставимый порядок, указанные показатели были статистически значимо ниже соответствующих среднероссийских данных (федеральный регистр СД, 2018 г.) - 52,2\% ( $\left.x^{2}=24,33 ; p<0,001\right)$ и 29,4\% ( $\left.X^{2}=23,99 ; p<0,001\right)$ (регистр СД, 2018 г. [1]).

Таблица 3. Характеристика сахароснижающей терапии, абс./\%

\begin{tabular}{|c|c|c|c|c|}
\hline \multirow{2}{*}{ Терапия } & \multicolumn{4}{|c|}{ Возрастная группа } \\
\hline & 45 лет, $N=33$ & 45-59 лет, $N=265$ & 60-74 года, $\mathrm{N}=697$ & >75-90 лет, $\mathrm{N}=207$ \\
\hline Немедикаментозная терапия & - & $4 / 1,51$ & $28 / 4,02$ & $35 / 16,91$ \\
\hline Неинсулиновые препараты & $26 / 78,79$ & $182 / 68,68$ & $486 / 69,72$ & $118 / 57,00$ \\
\hline Монотерапия & $19 / 57,58$ & $125 / 47,17$ & $401 / 57,53$ & $105 / 50,72$ \\
\hline 2 ССП & $9 / 27,27$ & $100 / 37,74$ & $192 / 27,55$ & $27 / 13,04$ \\
\hline 3 ССП & - & $14 / 5,28$ & $15 / 2,15$ & $3 / 1,45$ \\
\hline метформин & $25 / 75,76$ & $230 / 86,79$ & $579 / 83,07$ & $83 / 40,10$ \\
\hline ПСМ & $6 / 18,18$ & $87 / 32,93$ & $187 / 26,83$ & $68 / 32,85$ \\
\hline иДПП-4 & $3 / 9,09$ & $29 / 94$ & $42 / 6,03$ & $15 / 7,25$ \\
\hline глифлозины & $3 / 9,09$ & $20 / 7,54$ & $20 / 2,87$ & $1 / 0,48$ \\
\hline АрГПП-1 & - & $1 / 0,38$ & $1 / 0,14$ & $1 / 0,48$ \\
\hline Инсулинотерапия & $7 / 21,21$ & $79 / 29,81$ & $183 / 26,25$ & $54 / 26,09$ \\
\hline Монотерапия & $4 / 12,12$ & $22 / 8,30$ & $61 / 8,75$ & $36 / 17,39$ \\
\hline+1 ССП & $2 / 6,06$ & $30 / 11,32$ & $87 / 12,48$ & 16,73 \\
\hline+2 ССП & - & $26 / 9,81$ & $33 / 4,73$ & $2 / 0,97$ \\
\hline+3 ССП & - & $1 / 0,38$ & $2 / 0,29$ & - \\
\hline+ ПСМ & $1 / 3,03$ & $22 / 8,30$ & $33 / 4,73$ & $9 / 4,35$ \\
\hline Базис-болюсная терапия & $3 / 9,09$ & $43 / 16,23$ & $80 / 11,47$ & $16 / 7,73$ \\
\hline Смешанные инсулины & $2 / 6,06$ & $11 / 4,15$ & $59 / 8,46$ & $23 / 11,11$ \\
\hline ИКД/ИУКД & - & - & $1 / 0,14$ & - \\
\hline иСД/ИДД/ИУДД & $2 / 6,06$ & $15 / 5,66$ & $44 / 6,31$ & $15 / 7,25$ \\
\hline
\end{tabular}

Примечания: ССП — сахароснижающие препараты; идПП-4 - ингибиторы дипептидилпептидазы 4 типа; АрГпП-1 - агонисты рецепторов глюкагоноподобного пептида 1; ПСМ — препараты сульфонилмочевины; ИКД - инсулин короткого действия; ИУКД - инсулин ультракороткого действия; ИСД - инсулин средней длительности действия; ИДД - инсулин длительного действия; ИУДД - инсулин ультра- или сверхдлительного действия. 
Таблица 4. Характеристика достигнутого уровня гликированного гемоглобина в разных возрастных группах

\begin{tabular}{|c|c|c|c|c|}
\hline \multirow{2}{*}{ Показатель } & \multicolumn{4}{|c|}{ Возрастная группа } \\
\hline & $<45$ лет, $N=33$ & 45-59 лет, $N=265$ & 60-74 года, $\mathrm{N}=697$ & >75-90 лет, $N=207$ \\
\hline $\mathrm{HbA}_{1 c^{\prime}} \%, \mathrm{Me}(\mathrm{LQ} ; \mathrm{UQ})$ & $7,50(6,70 ; 8,40)$ & $7,50(6,50 ; 9,25)$ & $7,00(6,40 ; 8,30)$ & $6,90(6,40 ; 8,00)$ \\
\hline $\mathrm{HbA}_{1 \mathrm{c}}<6,0 \%$, абс. $/ \%$ & $1 / 3,03$ & $16 / 6,04$ & $57 / 8,18$ & $23 / 11,11$ \\
\hline $\mathrm{HbA}_{1 \mathrm{c}} 6-6,5 \%$, абс./\% & $2 / 6,06$ & $41 / 15,47$ & $125 / 17,93$ & $31 / 14,98$ \\
\hline $\mathrm{HbA}_{1 \mathrm{c}} 6,5-7,0 \%$, абс./\% & $10 / 30,30$ & $45 / 16,98$ & $140 / 20,09$ & $51 / 24,64$ \\
\hline $\mathrm{HbA}_{1 \mathrm{c}} 7,0-7,5 \%$, абс./\% & $3 / 9,09$ & $30 / 11,32$ & $102 / 14,63$ & $26 / 12,56$ \\
\hline $\mathrm{HbA}_{1 \mathrm{c}} 7,5-8,0 \%$, абс./\% & $4 / 12,12$ & $25 / 9,43$ & $63 / 9,04$ & $23 / 11,11$ \\
\hline $\mathrm{HbA}_{1 \mathrm{c}} 8,0-8,5 \%$, абс./\% & $5 / 15,15$ & $16 / 6,04$ & $47 / 6,74$ & $12 / 5,79$ \\
\hline $\mathrm{HbA}_{1 \mathrm{c}}>8,5 \%$, aбc. $/ \%$ & $8 / 24,24$ & $92 / 34,72$ & $163 / 23,39$ & $41 / 19,81$ \\
\hline
\end{tabular}

При оценке достигнутого уровня $\mathrm{HbA}_{1 с}$ указанный показатель находился выше индивидуального целевого уровня в 43,34\% случаев $(\mathrm{n}=521)$. Уровень $\mathrm{HbA}_{1 c}<6,5 \%$ регистрировался в четверти случаев (n=296; 24,62\%),

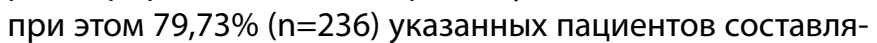
ли пациенты старшего возраста (60 лет и старше). Характеристика уровня $\mathrm{HbA}_{1 c}$ в различных возрастных группах подробно представлена в табл. 4.

Обращает на себя внимание то, что в общей группе пациентов в 97 случаях (8,07\%) HbA ставило 32,77\% наблюдений $\mathrm{HbA}_{1 c}<6,5 \%$. Вопреки ожидаемому, в общей группе уровень $\mathrm{HbA}_{1 c}$ не обнаружил существенных связей с возрастом пациентов и длительностью заболевания ( $\eta=-0,105$ и $\eta=0,178 ; p<0,05)$, такая же закономерность была выявлена среди пациентов c HbA 1 < $<7,0 \%$ ( $\eta=-0,059$ и $\eta=-0,103 ; p>0,05)$, с HbA 1 < $<6,5 \%$

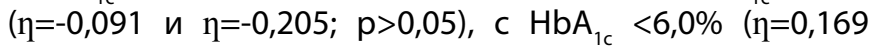
и r=-0,170; p>0,05). В общей группе также не было выявлено клинически значимых связей между уровнем $\mathrm{HbA}_{1 c}$ и полом пациентов ( $\eta=0,127 ; p<0,05)$, применением инсулинотерапии ( $\eta=0,318 ; \mathrm{p}<0,05)$, в том числе базис-болюсной инсулинотерапии ( $\eta=0,210 ; \mathrm{p}<0,05)$, неинсулиновой моно- $(\eta=-0,267 ; p<0,05)$ или комбинированной терапии $(\eta=0,183 ; p<0,05)$, применением ПСМ и/или инсулинотерапии $(\eta=0,411 ; p<0,05)$. В общей группе пациентов вклад всех перечисленных факторов в формирование результирующего признака «НbA 1 " составил 47,8\%, что предполагает наличие других влияющих факторов.

Уровень $\mathrm{HbA}_{1 с}$ (\%) среди пациентов, получающих ПСМ и/или инсулин, составил 8,00\% (LQ 7,00; UQ 9,30) и значимо отличался от показателя пациентов, получающих прочие препараты (6,60; LQ 6,20; UQ 7,20; U; p<0,001). В то же время среди пациентов, получающих ПСМ и/или инсулин, В сравнении с пациентами с другой сахароснижающей терапией значимо реже регистрировался уровень $\mathrm{HbA}_{1 с}<7 \%$ (25,59\% и 66,61\% соответственно; $\left.X^{2}=210,54 ; p<0,001\right),<6,5 \%$ (11,39\% и 38,42\% соответственно; $\left.X^{2}=113,19 ; p<0,001\right),<6,0 \%(4,95 \%$ и $11,58 \%$ соответственно; $\left.\chi^{2}=43,298 ; p=0,001\right)$, чаще - уровень $\mathrm{HbA}_{1 c}>8,5 \%$ (40,59\% и 10,57\% соответственно; $\left.x^{2}=157,56 ; p=0,001\right)$.

Среди пациентов, получающих базис-болюсный режим инсулинотерапии, $\mathrm{HbA}_{1 c}$ составил 8,30\% (LQ 7,10; UQ 9,80), значимо отличаясь от показателя остальных пациентов (7,00\% (LQ 6,40; UQ 8,20; U; p<0,001). Пациенты, получающие базис-болюсную терапию, также значимо отличались большей частотой регистрации $\mathrm{HbA}_{1 c}>8,5 \%$
(47,89\% и 122,17\% соответственно; $\left.X^{2}=46,231 ; p<0,001\right)$, меньшей частотой регистрации $\mathrm{HbA}_{1 c}<6,5 \%(9,15 \%$ и $26,51 \%$ соответственно; $\left.\mathrm{X}^{2}=17,154 ; \mathrm{p}=0,001\right), \mathrm{HbA}_{1 с}<7,0 \%$ $\left(17,61 \%\right.$ и 48,19\% соответственно; $\left.X^{2}=44,718 ; p<0,001\right)$, не выделяясь частотой $\mathrm{HbA}_{1 c}<6,0 \%(7,75 \%$ и $8,02 \%$ соответственно; $\left.x^{2}=0,005 ; p=1,000\right)$.

Оценка $\mathrm{HbA}_{1 c}$ в возрастных группах продемонстрировала наличие значимых отличий $(\mathrm{H} ; \mathrm{p}=0,004)$ : возраст $<45$ лет - $\mathrm{HbA}_{1 с} 7,5 \%$ (LQ 6,7; UQ 8,4); возраст 45-59 лет $\mathrm{HbA}_{1 \mathrm{c}} 7,5 \%$ (LQ 6,5; UQ 9,25); возраст 60-74 года - $\mathrm{HbA}_{1 \mathrm{c}}$ 7,0\% (LQ 6,4; UQ 8,3); возраст $\geq 75$ лет - HbA 1 6,9\% (LQ 6,4; UQ 8,0). Однако различия касались только уровня $\mathrm{HbA}_{1 c}$ в отдельных категориях: у пациентов в возрастных категориях «60-74» и « $\geq 75 » \mathrm{HbA}_{1 с}$ был значимо ниже, чем у пациентов среднего возраста $(U=8023,50 ; \mathrm{p}=0,002$ и $\mathrm{U}=2293,5 ; p=0,002$ соответственно).

При сравнении уровня $\mathrm{HbA}_{1 с}$ в старшей возрастной группе (пожилой возраст и старше) и в группе более молодого возраста было отмечено, что в старшей возрастной группе уровень $\mathrm{HbA}_{1 c^{\prime}}$ превышающий 8,5\%, регистрировался в пятой части случаев $(22,57 \%, \mathrm{n}=204)$, а среди пациентов молодого и среднего возраста в трети наблюдений (33,56\%; $\left.n=100 ; X^{2}=13,348 ; p<0,001\right)$. Следует отметить, что в старшей возрастной группе значимо чаще встречались пациенты с атеросклеротическими ССЗ (АССЗ) и/или ХБП 3-5 стадий в анамнезе, чем среди пациентов молодого и среднего возраста ( $\mathrm{n}=394 ; 43,58 \%$ и $\mathrm{n}=58 ; 19,46 \%$ соответственно; $\mathrm{x}^{2}=53,339 ;$ $\mathrm{p}=0,001) . \mathrm{HbA}_{1 c}<7 \%$ встречался в $47,23 \%(\mathrm{n}=427)$ случаев В старшей возрастной группе и в 38,59\% $(n=115)$ среди более молодых пациентов $\left(x^{2}=6,718 ; p=0,01\right)$. Среди пациентов старшего возраста с $\mathrm{HbA}_{1 c}<7,0 \%$ каждый пятый получал комбинированную неинсулиновую сахароснижающую терапию ( $\mathrm{n}=237 ; 19,72 \%)$, каждый третий $(\mathrm{n}=121$; $28,34 \%)$ получал ПСМ и/или инсулин, 38,64\% ( $n=165)$ имели АССЗ и/или ХБП 3-5 стадий.

Вопреки ожидаемому, уровень $\mathrm{HbA}_{1 c}<6,5 \%$, отражающий жесткий контроль гликемии, не выявил статистически значимых различий между группами (табл. 5). Указанный уровень $\mathrm{HbA}_{1 c}$ регистрировался у каждого пятого пациента молодого возраста и примерно у каждого четвертого пациента старшей возрастной группы. Таким образом, была обнаружена тенденция к более высокой частоте $\mathrm{HbA}_{1 c}<6,5 \%$ среди пациентов старшей возрастной группы, не достигшая, однако, статистической значимости $(p=0,067)$. 
Таблица 5. Частота выявления уровня гликированного гемоглобина ниже 6,5\% и ниже 6,0\% в основных возрастных группах

\begin{tabular}{|c|c|c|c|}
\hline \multirow[b]{2}{*}{ Показатель, абс./\% } & \multicolumn{2}{|c|}{ Возрастная группа } & \multirow[b]{2}{*}{$x^{2} ; p$} \\
\hline & $\begin{array}{c}\text { молодой } \\
\text { и средний, } \mathrm{N}=298\end{array}$ & $\begin{array}{c}\text { пожилой } \\
\text { и старше, N=904 }\end{array}$ & \\
\hline $\mathrm{HbA}_{1 \mathrm{c}}<6,5 \%$ & $60 / 20,13$ & $236 / 26,11$ & $x^{2}=3,343 ; p=0,067$ \\
\hline Атеросклеротические заболевания/ХБП & $12 / 20,00$ & $98 / 41,53$ & $x^{2}=7,759 ; p=0,006$ \\
\hline ПСМ и/или инсулин & $11 / 18,33$ & $58 / 24,58$ & $X^{2}=0,439 ; p=0,508$ \\
\hline Комбинированная неинсулиновая терапия & $10 / 16,67$ & $26 / 11,02$ & $X^{2}=0,291 ; p=0,590$ \\
\hline Базис-болюсная инсулинотерапия & $5 / 8,33$ & $10 / 4,24$ & $x^{2}=0,226 ; p=0,635$ \\
\hline $\mathrm{HbA}_{1 \mathrm{c}}<6,0 \%$ & $17 / 5,70$ & $80 / 8,85$ & $X^{2}=2,076 ; p=0,150$ \\
\hline Атеросклеротические заболевания/ХБП & $4 / 23,53$ & $39 / 48,75$ & $x^{2}=2,665 ; p=0,103$ \\
\hline ПСМ и/или инсулин & $6 / 35,29$ & $24 / 30,00$ & $x^{2}=0,118 ; p=0,732$ \\
\hline Комбинированная неинсулиновая терапия & $3 / 17,65$ & $10 / 12,50$ & $X^{2}=0,031 ; p=0,863$ \\
\hline Базис-болюсная инсулинотерапия & $3 / 17,65$ & $9 / 11,25$ & $X^{2}=1,251 ; p=0,264$ \\
\hline
\end{tabular}

Обращает на себя внимание то, что при уровне $\mathrm{HbA}_{1 с}$ $<6,5 \% 41,53 \%$ пациентов в возрасте 65 лет и старше имели АССЗ и/или ХБП, четверть пациентов (24,58\%) получали препараты, характеризующиеся повышенным риском гипогликемии. Среди пациентов этой возрастной группы с указанным уровнем $\mathrm{HbA}_{1 с} 19,07 \%$ ( $\left.\mathrm{n}=45\right)$, имея АCC3 и/или ХБП 3-5 стадии, получали в качестве сахароснижающей терапии ПСМ и/или инсулин. Подобная клиническая ситуация в группе пациентов более молодого возраста встречалась реже $\left(n=5 ; 8,33 \% ; x^{2}=3,199 ; p=0,074\right)$, причем $3 / 5$ пациентов имели $\mathrm{HbA}_{1 c}<6,0 \%$.

\section{ОБСУЖДЕНИЕ}

По данным исследования, в группе из 1202 пациентов с СД2 уровень $\mathrm{HbA}_{1 с}$ составил 7,1\% (LQ 6,5; UQ 8,5). У половины пациентов ( $\mathrm{n}=602 ; 50,08 \%)$ регистрировался уровень $\mathrm{HbA}_{1 с}$ в 2 крайних диапазонах значений, в соотношении 1:1 ( $<6,5 \%$ и >8,5\%). При оценке достигнутого уровня $\mathrm{HbA}_{1 с}$ относительно целевого уровня, он был выше целевого диапазона - в 43,34\% случаев $(n=521)$, $\mathrm{HbA}_{1 c}<7 \%$ зарегистрирован в $45,09 \%$ случаев, а уровень 7-7,9\% - у 22,96\% пациентов. Имея сопоставимый порядок, указанные показатели были статистически значимо ниже соответствующих среднероссийских данных (Федеральный регистр СД, 2018 г.). Не установлено связей клинически значимой силы уровня $\mathrm{HbA}_{1 с}$ с полом, возрастом пациентов, длительностью заболевания и особенностями терапии. С достаточно высокой частотой регистрировался жесткий контроль гликемии: уровень $\mathrm{HbA}_{1 c}<6,5 \%$ отмечался в четверти случаев $(24,62 \%)$, в том числе $<6,0 \%$ он был в 97 случаях, что составило треть всех случаев жесткого контроля гликемии. При этом большинство случаев $\mathrm{HbA}_{1 c}<6,5 \%$ приходилось на пациентов 60 лет и старше (79,73\%). В старшей возрастной группе достоверно реже регистрировался уровень, превышающий $8,5 \%(p<0,05)$, значимо чаще выявлялся $\mathrm{HbA}_{1 c}<7 \%(p<0,05)$, имелась тенденция к большей частоте $\mathrm{HbA}_{1 c}<6,5 \%$, отражающей жесткий контроль гликемии $(p=0,067)$. В последнем случае среди пациентов в $41,53 \%$ наблюдений имелись данные о наличии АССЗ и/или ХБП 3-5 стадий, в четверти случаев в терапии применялись
ПСМ и/или инсулин (24,58\%), практически каждый пятый пациент, имея атеросклеротическое заболевание и/или ХБП 3-5 ст., получал ПСМ и/или инсулин.

Многие исследования показали, что жесткий гликемический контроль, принося некоторые преимущества в отношении диабетической ретинопатии, неэффективен в отношении конечных сердечно-сосудистых точек и может быть связан с неблагоприятными событиями, связанными с гипогликемическими последствиями, особенно у пожилых пациентов [7-13]. Принятая Американской диабетической ассоциацией, Европейской ассоциацией по изучению диабета, Российской ассоциацией эндокринологов позиция по персонализации целевого уровня $\mathrm{HbA}_{1 с}$ в диапазоне от $<6,5 \%$ до $<8,5 \%$ подразумевает более свободные цели терапии в пожилом возрасте, основанные на оценке риска гипогликемии, наличия заболеваний атеросклеротического генеза, старческой астении и социальной адаптации $[2,14,15]$. В связи с этим в течение последних лет сформировалось понимание избыточности лечения пациентов с СД (сахароснижающей, гипотензивной терапии) как варианта неадекватного контроля заболевания, не уступающего по своей клинической значимости отсутствию достижения соответствующих целевых значений.

По данным зарубежных авторов, чрезмерное лечение, определяемое как лечение в случаях, когда потенциальный вред превышает возможную пользу, является распространенным явлением в практической медицине, помимо негативных клинических последствий, существенно повышающим затраты здравоохранения [7].

Как справедливо замечено, достижение "жесткого» гликемического контроля СД не является самоцелью и может свидетельствовать об избыточности терапии, если пациенты, достигшие $\mathrm{HbA}_{1 с}<7 \%$, имеют минимальную потенциальную пользу, повышенный риск негативных последствий и/или продолжительность жизни короче, чем период времени, необходимый для получения клинических преимуществ [7]. По данным Lipska и соавт., в США около 60\% пациентов пожилого возраста с СД2 с ограниченной продолжительностью жизни имеют $\mathrm{HbA}_{1 c}<7 \%$, в $60 \%$ случаев получая инсулин /или ПСМ [4]. С другой стороны, по некоторым данным, до 50\% пациентов, получающих инсулин и/или ПСМ, в возрасте 75 лет и старше 
имеют $\mathrm{HbA}_{1 c}<7 \%$ [16]. По данным исследователей из США, именно такие пациенты имеют наибольший риск госпитализаций по поводу гипогликемии [17]. В нашем исследовании были получены сопоставимые данные: уровень $\mathrm{HbA}_{1 c}$ менее 7\% в старшей возрастной группе был зарегистрирован у 47,57\% пациентов, среди которых примерно каждый третий получал ПСМ и/или инсулин (28,34\%), каждый пятый — комбинированную неинсулиновую сахароснижающую терапию (19,72\%). Кроме того, по нашим данным, 38,64\% пациентов пожилого и старческого возраста с HbA 1 < $<\%$ имели АССЗ и/или ХБП 3-5 стадий.

Следует отметить, что в зарубежных исследованиях критерии избыточной терапии разнятся. Так, в турецком исследовании за чрезмерное лечение принимали $\mathrm{HbA}_{1 c}$ $<6,5 \%$ в сочетании с использованием 2 и более пероральных сахароснижающих препаратов или инсулина. Среди пациентов пожилого возраста (65 лет и старше) указанные критерии были выполнены для 9,8\% пациентов [18]. В нашем наблюдении среди лиц пожилого возраста и старше примерно каждый четвертый пациент (26,11\%) имел уровень $\mathrm{HbA}_{1 c}<6,5 \%$, из них 24,58\% получили ПСМ и/или инсулин, 11,02\% - комбинированную неинсулиновую терапию. Более того, практически каждый десятый пациент (8,85\%) из старшей возрастной группы имел $\mathrm{HbA}_{1 c}<6,0 \%$.

Целью сахароснижающей терапии при СД2 является снижение риска развития осложнений СД при минимизации вреда, связанного с терапией, и, таким образом, увеличение как продолжительности жизни, так и качества жизни, связанного со здоровьем. Данные о смертности в ACCORD продемонстрировали, что потенциальные риски интенсивного гликемического контроля $\left(\mathrm{HbA}_{1 c}<6,0 \%\right)$ могут перевешивать его преимущества у пациентов с более высоким сердечно-сосудистым риском [19]. Отдаленные результаты исследования ADVANCE (целевой $\mathrm{HbA}_{1 c}$ $<6,5 \%)$, хотя и не выявили вреда, но также не выявили и сердечно-сосудистых преимуществ интенсивного контроля гликемии, что также актуализирует вопрос о рациональности лекарственной нагрузки, особенно в группе пациентов высокого риска [20]. Метаанализы исследований интенсивного гликемического контроля показывают, что снижение уровня глюкозы может вызывать скромное, но статистически значимое уменьшение основных исходов СС3, в первую очередь нефатального инфаркта миокарда, но не оказывает существенного влияния на смертность [21-24]. Однако такое преимущество снижения уровня глюкозы при СС3 незначительно по сравнению с коррекцией других факторов риска ССЗ [25]. В связи с этим определение целевого уровня $\mathrm{HbA}_{1 с}$ действительно должно быть взвешенным и максимально индивидуализированным.

Установление факта неадекватности терапии, безусловно, преследует цель выявления группы пациентов, нуждающихся в ее коррекции. Деинтенсификация терапии - следующий шаг в тактике ведения пациентов с избыточностью лечения, имеющий важное клинико-экономическое значение. С одной стороны, обоснованная деинтенсификация терапии снижает прямые расходы на лечение. С другой стороны, устраняя полипрагмазию, позволяет предотвратить ее значимые клинические последствия. Для сахароснижающих препаратов это прежде всего гипогликемия и ее последствия.
Причисляя пациентов с избыточным снижением $\mathrm{HbA}_{1 с}$ в группу риска по развитию гипогликемии, следует подчеркнуть, что с клинической точки зрения $\mathrm{HbA}_{1 с}$ отнюдь не является надежным маркером указанного риска. Взаимосвязь между риском гипогликемии и интенсивностью лечения существенно отличается от взаимосвязи между риском гипогликемии и достигнутым уровнем $\mathrm{HbA}_{1 c}$. В ряде исследований показано, что по крайней мере частота «самозаявленных» (self-reported) тяжелых гипогликемий либо ассоциирована с повышением HbA1c, либо от него не зависит $[17,26]$. Тем не менее снижение $\mathrm{HbA}_{1 c}$ до уровня, отражающего жесткий контроль гликемии, всегда следует рассматривать с точки зрения преимуществ и недостатков, соответственно, необходимости деинтенсификации терапии. Эксперты подчеркивают, что достижение $\mathrm{HbA}_{1 c}<6,5 \%$ возможно лишь при условии его безопасности, отсутствия гипогликемии или других побочных эффектов [14]. Принципиально важно выделять пациентов, имеющих потенциальный риск гипогликемии, ассоциированный с сахароснижающими средствами, в частности инсулином и ПСМ. В связи с этим как минимум четверть пациентов пожилого возраста и старше, имеющих $\mathrm{HbA}_{1 c}<6,5 \%$ и получающих СМ и/или инсулин, представляют собой группу, однозначно нуждающуюся в деинтенсификации терапии. На наш взгляд, при прочих равных условиях это именно та группа больных СД2, которая нуждается в скорейшей деинтенсификации терапии. Безусловно, настораживающим является факт, что именно среди пациентов старшей возрастной группы, по данным регистра, чаще отмечается избыточность терапии, косвенно свидетельствуя о несвоевременности принятия клинического решения. Это наблюдение вполне соотносится с данными зарубежных коллег, демонстрирующими, что деинтенсификация сахароснижающей терапии в старшей возрастной группе проводится лишь в четверти случаев [18, 27].

Безусловно, следует отметить, что в старшей возрастной группе в качестве желательных обсуждаются более высокие уровни $\mathrm{HbA}_{1 c}$. При этом, в отличие от отечественных клинических рекомендаций, рассматриваются уровни $\mathrm{HbA}_{1 c}$ с указанием нижней границы рекомендованного диапазона. В японских клинических рекомендациях, обозначающих нижнюю границу целевого диапазона гликемии для трех категорий пациентов старшего возраста с различными характеристиками функциональных возможностей и наличием/отсутствием старческой астении: $<7,5 \%$ (нижняя граница 6,5\%), <8\% (нижняя граница 7,0\%), <8,5\% (нижняя граница 7,5\%) [28].

В рекомендациях других экспертных сообществ обозначаются более высокие нижние границы диапазонов $\mathrm{HbA}_{1 с}$ для пациентов старшего возраста. В частности, экспертная группа Американского общества гериатров так обозначила целевой уровень $\mathrm{HbA}_{1 с}$ для старшей возрастной группы:

целевой уровень у пожилых пациентов обычно должен составлять от 7,5 до 8,0\% (уровень доказательств 1A);

$\mathrm{HbA}_{1 с}$ между 7,0 и 7,5\% может быть приемлемым, если может быть безопасно достигнут у здоровых пожилых пациентов с небольшим количеством сопутствующих заболеваний и хорошим функциональным статусом; 
- $\mathrm{HbA}_{1 c}$ 8,0-9,0\% подходит для пожилых людей с множественными сопутствующими заболеваниями, плохим здоровьем и ограниченной ожидаемой продолжительностью жизни (уровень доказательств ІІА);

- существует потенциальный вред в снижении $\mathrm{HbA}_{1 с}$ до $<6,5 \%$ у пожилых пациентов с СД 2 типа [29].

Рекомендации эндокринологического общества по ведению диабета в старшей возрастной группе (65 лет и старше) уточняют не только клинический и функциональный статус пациентов, но и характер терапии. Так, при применении инсулина и/или ПСМ, глинидов обозначаются целевые диапазоны $\mathrm{HbA}_{1 с}$ для 3 категорий пациентов ( 1 - здоровые; 2 - промежуточная категория; 3 - плохое состояние здоровья): $\geq 7,0-<7,5 \% ; \geq 7,5-<8,0 \%$ и $\geq 8,0-<8,5 \%$ [30]. Исходя из подобного подхода, в нашем наблюдении группа пациентов, нуждающихся в деинтенсификации текущей терапии, может быть еще более широкой.

$\mathrm{HbA}_{1 с}$ является косвенным показателем среднего уровня гликемии и имеет известные ограничения (наличие гемоглобинопатий, анемии, терминальная стадия ХБП, дефицит глюкозо-6-фосфатдегидрогеназы и пр.). Кроме того, как известно, $\mathrm{HbA}_{1 с}$ не обеспечивает оценки вариабельности гликемии или гипогликемии. В связи с этим полученные нами данные по различиям частоты регистрации $\mathrm{HbA}_{1 c}<6,0,<6,5,<7,0,>8,5 \%$ между пациента- ми, получающими и не получающими ПСМ и/или инсулин, базис-болюсную инсулинотерапию, трудно трактовать однозначно. Однако с клинической точки зрения, безусловно, особое внимание следует обращать именно на $\mathrm{HbA}_{1 c^{\prime}}$ результат которого близок к порогу, который может побудить к изменению медикаментозной терапии [14].

Принимая во внимание объективную ограниченность трактовки $\mathrm{HbA}_{1 c^{\prime}}$ рекомендации Американской диабетической ассоциации по целевому $\mathrm{HbA}_{1 c^{\prime}}$ дополненные желательным диапазоном гликемии, выглядят заслуживающими внимания, особенно в группе пациентов старшего возраста (табл. 6). Так, в группе взрослых пациентов вне беременности определяются следующие показатели: целевой $\mathrm{HbA}_{1 c}<7,0 \%$, целевая препрандиальная глюкоза капиллярной крови 4,4-7,2 ммоль/л, целевая пиковая постпрандиальная глюкоза капиллярной крови $<10,0$ ммоль/л. В то же время для пациентов старшей возрастной группы рекомендации включают такие параметры, как «глюкоза крови натощак и препрандиально» и «глюкоза крови перед сном». В рекомендациях выделяются три категории пациентов старшего возраста по признакам наличия и выраженности сопутствующих хронических заболеваний, состояния когнитивного и функционального статуса с различными характеристиками длительности жизни (см. табл. 6).

Таблица 6. Объединенное резюме рекомендаций по контролю гликемии в старшей возрастной группе пациентов (ADA, 2020), цитируется по [14]

\begin{tabular}{|c|c|c|c|c|}
\hline \multirow[b]{2}{*}{ Группа пациентов } & \multirow[b]{2}{*}{ Обоснование } & \multicolumn{3}{|c|}{ Целевой уровень } \\
\hline & & $\mathrm{HbA}_{1 \mathbf{c}^{\prime}} \%$ & $\begin{array}{c}\text { глюкоза крови } \\
\text { натощак и } \\
\text { препрандиально, } \\
\text { ммоль/л }\end{array}$ & $\begin{array}{c}\text { глюкоза } \\
\text { крови } \\
\text { перед сном, } \\
\text { ммоль/л }\end{array}$ \\
\hline $\begin{array}{l}\text { Здоровые (несколько сосуществующих } \\
\text { хронических заболеваний, } \\
\text { сохраненный когнитивный } \\
\text { и функциональный статусы) }\end{array}$ & $\begin{array}{l}\text { Более длинная } \\
\text { ожидаемая } \\
\text { продолжительность } \\
\text { жизни }\end{array}$ & $<7,5$ & $5,0-7,2$ & $5,0-8,3$ \\
\hline $\begin{array}{l}\text { Сложные / промежуточные } \\
\text { характеристики (множественные } \\
\text { сосуществующие хронические } \\
\text { заболевания*, или 2+ нарушений } \\
\text { инструментальных действий } \\
\text { по удовлетворению повседневных } \\
\text { нужд, или когнитивные нарушения } \\
\text { легкой и средней степени тяжести) }\end{array}$ & $\begin{array}{l}\text { Промежуточная } \\
\text { ожидаемая } \\
\text { продолжительность } \\
\text { жизни, высокая } \\
\text { лекарственная нагрузка, } \\
\text { подверженность } \\
\text { гипогликемии, риск } \\
\text { падения }\end{array}$ & $<8,0$ & $5,0-8,3$ & $5,6-10,0$ \\
\hline $\begin{array}{l}\text { Очень сложные / плохое здоровье } \\
\text { (потребность в долгосрочном уходе, } \\
\text { или терминальная стадия хронических } \\
\text { заболеваний }{ }^{* *} \text {, или умеренные или } \\
\text { тяжелые когнитивные нарушения, } \\
\text { или зависимость от 2+ нарушений } \\
\text { инструментальных действий } \\
\text { по удовлетворению повседневных нужд) }\end{array}$ & $\begin{array}{l}\text { Ограниченная } \\
\text { ожидаемая } \\
\text { продолжительность } \\
\text { жизни делает выгоды } \\
\text { неопределенными }\end{array}$ & $<8,5 \%$ & $5,6-10,0$ & $6,1-11,1$ \\
\hline
\end{tabular}

Примечание: * Сосуществующие хронические заболевания - состояния достаточно серьезные, чтобы требовать медикаментозного лечения или изменения образа жизни, могут включать артрит, рак, застойную сердечную недостаточность, депрессию, эмфизему, падения, артериальную гипертензию, недержание мочи, ХБП 3-6 стадий, инфаркт миокарда и инсульт. «Множественные» - по крайней мере три, пациенты могут иметь пять или более; ** Наличие одной терминальной стадии хронического заболевания, например стадии 3-4 ХСН или кислородзависимое заболевание легких; ХБП, требующая диализа, или неконтролируемый метастатический рак могут вызывать значительные симптомы или нарушения функционального состояния, значительно сокращают продолжительность жизни.

' - Уровень $\mathrm{HbA}_{1 c}$ 8,5\% приравнивается к предполагаемой средней глюкозе 11,1 ммоль/л. Цели выше 8,5\% не рекомендуются, так как они могут подвергать пациентов риску более частого и более высокого уровня глюкозы, рискам, связанным с глюкозурией, дегидратацией, риску развития гипергликемического гиперосмолярного синдрома, плохого заживления ран. 
Деинтенсификация терапии (синоним в зарубежной литературе - deprescribing) подразумевает снижение дозы препарата, переход на более безопасный препарат или прекращение приема лекарственного средства. По мнению канадских экспертов (врачи общей практики, эндокринологи, фармацевты), рекомендации по деинтенсификации терапии в старшей возрастной группе должны выполняться для пациентов старше 65 лет, принимающих $\geq 1$ антигипергликемических препаратов для лечения СД2 и отвечающих $\geq 1$ из следующих критериев:

- принадлежность к группе риска гипогликемии (вследствие преклонного возраста, чрезмерно интенсивного контроля гликемии, множественных сопутствующих заболеваний, лекарственных взаимодействий, гипогликемии в анамнезе или отсутствия распознавания гипогликемии, нарушения функции почек или приема ПСМ или инсулина);

- подверженность риску возникновения других побочных эффектов сахароснижающих препаратов;

- преимущества (польза) терапии неопределенны вследствие ослабленности, деменции или ограниченной продолжительности жизни [31].

При этом авторы рекомендаций совершенно справедливо обозначают необходимые клинические решения: деинтенсификация сахароснижающей терапии (deprescribing) препаратами, способствующими развитию гипогликемии; деинтенсификация терапии у пациентов, испытывающих или находящихся в группе риска неблагоприятных эффектов; индивидуализация гликемических целей и деинтенсификация терапии в соответствии с ними [31]. Мы полагаем, что во всех обозначенных нами случаях избыточности терапии требуется как минимум скорейшая дополнительная индивидуальная клиническая оценка, а в старшей группе пациентов деинтенсификация терапии.

В своей работе мы сконцентрировали внимание на пациентах старшей возрастной группы, как наиболее уязвимых для клинических последствий избыточной сахароснижающей терапии. Именно этой группе пациентов посвящены исследования и клинические рекомендации зарубежных коллег. Однако следует помнить, что понятия «избыточность» и «деинтенсификация» терапии универсальны и применимы к определенным клиническим ситуациям, которые возможны в любом возрасте. В отсутствие единого подхода еще сложнее обсуждать данную тему в группе пациентов более молодого возраста. В нашем исследовании жесткий контроль гликемии был достигнут, несмотря на наличие факторов риска тяжелой гипогликемии и одновременное применение ПСМ и/или инсулина у 8,33\% пациентов молодого и среднего возраста. Как правило, в реальной клинической практике в подобных случаях врачебное решение основывается прежде всего на переносимости терапии, т.е. наличии гипогликемии, в том числе эпизодов самозаявленной легкой гипогликемии. Однако следует помнить о феномене нарушения распознавания гипогликемии. Так, отсутствие и нарушение распознавания гипогликемии, по российским данным, встречается у пациентов с СД2 в 21,1 и 32,1\% случаев соответственно и не зависит от длительности диабета и инсулинотерапии как таковой [32]. Таким образом, несмотря на редкость регистрации среди лиц молодого и среднего возраста с СД2, ситуации избыточности терапии возможны и могут быть клинически недооценены.

В российских «Алгоритмах специализированной медицинской помощи больным СД» ясно обозначается необходимость снижения риска гипогликемии, тем не менее отсутствуют четкие рекомендации по их профилактике [2, 33]. Своевременная деинтенсификация лекарственной нагрузки как вариант оптимизации сахароснижающей терапии, безусловно, нуждается в полноценном внимании экспертов. В недавно опубликованном отечественном обзоре современной литературы, посвященной данной теме, авторами справедливо обозначены основные цели разработки концепции депрескрайбинга сахароснижающих препаратов: формирование четких показаний, дальнейшее изучение результатов депрескрайбинга сахароснижающих средств в клинических исследованиях; разработка алгоритма и промежуточных точек контроля его результатов; подготовка соответствующих клинических рекомендаций [33].

Ограничения исследования. Можно предполагать, что не все факторы, влияющие на клиническую трактовку $\mathrm{HbA}_{1 c}$ и упомянутые выше, были отражены в регистре. В связи с отсутствием в нем данных о функциональной активности и наличии/отсутствии старческой астении/ деменции эти параметры не учитывались при расчете целевого $\mathrm{HbA}_{1 c^{\prime}}$ что могло привести к занижению уровня целевого $\mathrm{HbA}_{1 c}$ и, соответственно, к недооценке распространенности избыточного снижения $\mathrm{HbA}_{1 с}$ среди больных с СД2 в старшей возрастной группе.

\section{ЗАКЛЮЧЕНИЕ}

Используя выборку пациентов СД2 из регионального регистра СД, мы показали, что уровень $\mathrm{HbA}_{1 с}<6,5 \%$ отмечается в четверти случаев (24,62\%), в том числе $\mathrm{HbA}_{1 с}$ $<6,0 \%$ был зарегистрирован в 97 случаях, что составило треть от всех случаев жесткого контроля гликемии. При этом большинство наблюдений $\mathrm{HbA}_{1 c}<6,5 \%$ приходилось на пациентов 60 лет и старше (79,73\%). В старшей возрастной группе (в сравнении с пациентами молодого и среднего возраста) значимо чаще выявлялся $\mathrm{HbA}_{1 с}$ $<7 \%(\mathrm{p}<0,05)$, имелась тенденция к большей частоте $\mathrm{HbA}_{1 c}<6,5 \%$, отражающей жесткий контроль гликемии $(p=0,067)$. Среди пациентов пожилого и старческого возраста, имевших $\mathrm{HbA}_{1 c}<6,5 \%$, в 41,53\% наблюдений имелись факторы риска тяжелой гипогликемии, в четверти случаев в терапии СД2 применялись ПСМ и/или инсулин (24,58\%), практически каждый пятый пациент (19,07\%), имея АССЗ и/или ХБП 3-5 ст., получал ПСМ и/или инсулин. Среди пациентов молодого и среднего возраста с $\mathrm{HbA}_{1 \mathrm{c}}<6,5 \%$ в 8,33\% случаев имелись факторы риска тяжелой гипогликемии при наличии терапии ПСМ и/или инсулином. По нашим данным, как минимум четверть пациентов старшей возрастной группы (24,58\%) нуждаются в деинтенсификации терапии. Принимая во внимание позицию некоторых экспертных сообществ по определению целевого диапазона $\mathrm{HbA}_{1 c}$ в старшей возрастной группе, потребность в деинтенсификации сахароснижающей терапии может быть еще более высокой. Требуются дальнейшие исследования с целью разработки полноценной отечественной концепции деинтенсификации сахароснижающей терапии. 


\section{ДОПОЛНИТЕЛЬНАЯ ИНФОРМАЦИЯ}

Финансирование работы. Работа проведена за счет бюджетных средств организаций.

Конфликт интересов. Авторы декларируют отсутствие явных и потенциальных конфликтов интересов, связанных с публикацией настоящей статьи.

Участие авторов. Друк И.В. - концепция и дизайн исследования, анализ полученных данных, написание текста; Снарская Д.И. - обработка материалов, анализ полученных данных; Горощеня О.И. - сбор и обработка материалов. Все авторы внесли значимый вклад в проведение исследования и подготовку статьи, прочли и одобрили финальную версию статьи перед публикацией.

Благодарности. Авторы выражают благодарность Родионовой Л.Ю. - главному специалисту-эндокринологу МЗ Омской области за техническую поддержку.

\section{СПИСОК ЛИТЕРАТУРЫ | REFERENCES}

1. Шестакова М.В., Викулова О.К., Железнякова А.В., и др., Эпидемиология сахарного диабета в Российской Федерации: что изменилось за последнее десятилетие? // Терапевтический архив. - 2019. — №10. - C. 4-13. [Shestakova MV, Vikulova OK, Zheleznyakova AV, et al. Diabetes epidemiology in Russia: what has changed over the decade? Therapeutic Archive. 2019;91(10):4-13. (In Russ.)]. doi: https://doi.org/10.26442/00403660.2019.10.000364

2. Дедов И.И., Шестакова М.В., Майоров А.Ю., и др. Алгоритмы специализированной медицинской помощи больным сахарным диабетом / Под ред. И.И. Дедова, М.В. Шестаковой, А.Ю. Майорова. 9-й выпуск // Сахарный диабет. - 2019. - Т. 22. - №S1-1. C. 1-144. [Dedov II, Shestakova MV, Mayorov AY, et al. Standards of specialized diabetes care. Ed. by Dedov II, Shestakova MV, Mayorov AY. 9th edition. Diabetes Mellitus. 2019;22(S1-1):1-144. (In Russ.)]. doi: https://doi.org/10.14341/DM221S1

3. Дедов И.И., Шестакова М.В., Викулова О.К., и др. Атлас регистра сахарного диабета Российской Федерации. Статус 2018 г. // Сахарный duабет. — 2019. — №22(2S). — C. 4-61. [Dedov II, Shestakova MV, Vikulova OK, et al. Atlas of the diabetes register of the Russian Federation. Status 2018. Diabetes Mellitus. 2019;22(2S):4-61. (In Russ.)]. doi: https://doi.org/10.14341/DM12208

4. Lipska KJ, Ross JS, Miao Y, et al. Potential overtreatment of diabetes mellitus in older adults with tight glycemic control. JAMA Intern Med. 2015;175(3):356-362. doi: https://doi.org/10.1001/jamainternmed.2014.7345

5. Sonmez A, Tasci I, Demirci I, et al. A Cross-Sectional Study of Overtreatment and Deintensification of Antidiabetic and Antihypertensive Medications in Diabetes Mellitus: The TEMD Overtreatment Study. Diabetes Ther. 2020;11(5):1045-1059. doi: https://doi.org/10.1007/s13300-020-00779-0

6. McAlister FA, Youngson E, Eurich DT. Treatment Deintensification Is Uncommon in Adults With Type 2 Diabetes Mellitus. Circ Cardiovasc Qual Outcomes. 2017;10(4). doi: https://doi.org/10.1161/CIRCOUTCOMES.116.003514

7. Makam AN, Nguyen OK. An Evidence-Based Medicine Approach to Antihyperglycemic Therapy in Diabetes Mellitus to Overcome Overtreatment. Circulation. 2017;135(2):180-195. doi: https://doi.org/10.1161/CIRCULATIONAHA.116.022622

8. McAlister FA, Youngson E, Eurich DT. Effects of Intensive Blood-Pressure Control in Type 2 Diabetes Mellitus. N Engl J Med. 2010;362(17):1575-1585. doi: https://doi.org/10.1056/NEJMoa1001286

9. McAlister FA, Youngson E, Eurich DT. Effects of Intensive Glucose Lowering in Type 2 Diabetes. N Engl J Med. 2008;358(24):2545-2559. doi: https://doi.org/10.1056/NEJMoa0802743

10. McAlister FA, Youngson E, Eurich DT. Intensive Blood Glucose Control and Vascular Outcomes in Patients with Type 2 Diabetes. N Engl J Med. 2008;358(24):2560-2572. doi: https://doi.org/10.1056/NEJMoa0802987

11. Duckworth W, Abraira C, Moritz T, et al. Glucose Control and Vascular Complications in Veterans with Type 2 Diabetes. N Engl J Med. 2009;360(2):129-139. doi: https://doi.org/10.1056/NEJMoa0808431

12. Hemmingsen B, Lund SS, Gluud C, et al. Intensive glycaemic control for patients with type 2 diabetes: systematic review with meta-analysis and trial sequential analysis of randomised clinical trials. BMJ. 2011;343(24-1):d6898-d6898. doi: https://doi.org/10.1136/bmj.d6898

13. Rodríguez-Gutiérrez R, Montori VM. Glycemic control for patients with type 2 diabetes mellitus: our evolving faith in the face of evidence. Circ Cardiovasc Qual Outcomes. 2016;9:504-512. doi: https://doi.org/10.1161/CIRCOUTCOMES.116.002901
14. American Diabetes Association. Standards of Medical Care in Diabetes-2020. Diabetes Care. 2020;43(1):S1-S212.

15. Davies MJ, D'Alessio DA, Fradkin J, et al. Management of hyperglycaemia in type 2 diabetes, 2018. A consensus report by the American Diabetes Association (ADA) and the European Association for the Study of Diabetes (EASD). Diabetologia. 2018;61(12):2461-2498. doi: https://doi.org/10.1007/s00125-018-4729-5

16. Tseng $\mathrm{CL}$, Soroka $\mathrm{O}$, Maney $\mathrm{M}$, et al. Assessing potential glycemic overtreatment in persons at hypoglycemic risk. JAMA Intern Med. 2014;174(2):259-268. doi: https://doi.org/10.1001/jamainternmed.2013.12963

17. Lipska KJ, Warton EM, Huang ES, et al. HbA1c and risk of severe hypoglycemia in type 2 diabetes: the Diabetes and Aging Study. Diabetes Care. 2013;36(11):3535-3542. doi: https://doi.org/10.2337/dc13-0610

18. Davis TM, Brown SG, Jacobs IG, et al. Determinants of severe hypoglycemia complicating type 2 diabetes: the Fremantle diabetes study. J Clin Endocrinol Metab. 2010;95(5):2240-2247. doi: https://doi.org/10.1210/jc.2009-2828

19. Gerstein HC, Miller ME, Byington RP, et al. Action to Control Cardiovascular Risk in Diabetes Study Group. Effects of intensive glucose lowering in type 2 diabetes. N Engl J Med. 2008;358:2545-2559. doi: https://doi.org/10.1056/NEJMoa0802743

20. Zoungas S, Chalmers J, Neal B, et al. ADVANCE-ON Collaborative Group. Follow-up of blood-pressure lowering and glucose control in type 2 diabetes. N Engl J Med. 2014;371:1392-1406. doi: https://doi.org/10.1056/NEJMoa1407963

21. Turnbull FM, Abraira C, Anderson RJ, et al. Intensive glucose control and macrovascular outcomes in type 2 diabetes. Diabetologia. 2009;52(11):2288-2298. doi: https://doi.org/10.1007/s00125-009-1470-0

22. Ray KK, Seshasai SRK, Wijesuriya S, et al. Effect of intensive control of glucose on cardiovascular outcomes and death in patients with diabetes mellitus: a meta-analysis of randomised controlled trials. Lancet. 2009;373(9677):1765-1772. doi: https://doi.org/10.1016/S0140-6736(09)60697-8

23. Kelly TN. Systematic Review: Glucose Control and Cardiovascular Disease in Type 2 Diabetes. Ann Intern Med. 2009;151(6):394-403. doi: https://doi.org/10.7326/0003-4819-151-6-200909150-00137

24. Boussageon R, Bejan-Angoulvant T, Saadatian-Elahi M, et al. Effect of intensive glucose lowering treatment on all cause mortality, cardiovascular death, and microvascular events in type 2 diabetes: meta-analysis of randomised controlled trials. BMJ. 2011;343(1):d4169-d4169. doi: https://doi.org/10.1136/bmj.d4169

25. Fox CS, Golden SH, Anderson C, et al. Update on Prevention of Cardiovascular Disease in Adults With Type 2 Diabetes Mellitus in Light of Recent Evidence: A Scientific Statement From the American Heart Association and the American Diabetes Association. Diabetes Care. 2015;38(9):1777-1803. doi: https://doi.org/10.2337/dci15-0012

26. Sonmez A, Tasci I, Demirci I, et al. A Cross-Sectional Study of Overtreatment and Deintensification of Antidiabetic and Antihypertensive Medications in Diabetes Mellitus: The TEMD Overtreatment Study. Diabetes Ther. 2020;11(5):1045-1059. doi: https://doi.org/10.1007/s13300-020-00779-0

27. Pirela DV, GARG R. 399-P: Deintensification of Diabetes Treatment in Elderly Patients with Type 2 Diabetes Mellitus. Diabetes. 2019;68(1):399-P. doi: https://doi.org/10.2337/db19-399-P

28. Araki E, Goto A, Kondo T, et al. Japanese Clinical Practice Guideline for Diabetes 2019. J Diabetes Investig. 2020;11(4):1020-1076. doi: https://doi.org/10.1111/jdi.13306 
29. Boussageon R, Bejan-Angoulvant T, Saadatian-Elahi M, et al. Guidelines Abstracted from the American Geriatrics Society Guidelines for Improving the Care of Older Adults with Diabetes Mellitus: 2013 Update. J Am Geriatr Soc. 2013;61(11):2020-2026. doi: https://doi.org/10.1111/jgs.12514

30. LeRoith D, Biessels GJ, Braithwaite SS, et al. Treatment of Diabetes in Older Adults: An Endocrine Society* Clinical Practice Guideline. J Clin Endocrinol Metab. 2019;104(5):1520-1574. doi: https://doi.org/10.1210/jc.2019-00198

31. Farrell B, Black C, Thompson W, et al. Deprescribing antihyperglycemic agents in older persons. Canadian Family Physician. 2017;63(11):832-843.

32. Климонтов В.В. Нарушение распознавания гипогликемии при сахарном диабете: эпидемиология, механизмы развития, терапевтические подходы // Сахарный duaбem - 2018. - T. 21 - №6. - C. 513-523. [Klimontov VV. Impaired recognition of hypoglycemia in diabetes mellitus: epidemiology, mechanisms of development, therapeutic approaches. Diabetes mellitus. 2018;21(6):513-523. (In Russ.)]. doi: https://doi.org/10.14341/DM9597

33. Ткачева О.Н., Остроумова О.Д., Котовская Ю.В., и др. Депрескрайбинг сахароснижающих препаратов у пациентов пожилого и старческого возраста: современное состояние вопроса и обзор доказательной базы // Клиническая фармакология и терапия. - 2019. — №10. - C. 4-13. [Tkacheva ON, Ostroumova OD, Kotovskaya YuV, et al. Deprescribing of hypoglycemic drugs in elderly and senile patients: current state of the issue and review of the evidence base. Clinical pharmacology and therapy. 2019;28(3):62-67. (In Russ.)]. doi: https://doi.org/10.32756/0869-5490-2019-3-62-67

\section{ИНФОРМАЦИЯ ОБ АВТОРАХ [AUTHORS INFO]}

*Друк Инна Викторовна, д.м.н., доцент [Inna V. Druk, MD, PhD, associate professor]; адрес: Россия, 644009, Омск, ул. Ленина, д. 12 [address: 12 Lenina street, 644009 Omsk, Russia]; телефон: +79139641555;

ORCID: https://orcid.org/0000-0001-8317-7765; eLibrary SPIN: 9069-1518; e-mail: drukinna@yandex.ru

Снарская Диана Ильдаровна [Diana I. Snarskaya]; ORCID: https://orcid.org/0000-0002-1733-802X; e-mail: dina.valieva.94654@mail.ru

Горощеня Оксана Ивановна [Oksana I. Goroshchenya]; ORCID: https://orcid.org/0000-0001-6856-5225; e-mail: goroshchenya67@mail.ru

\section{ЦИТИРОВАТЬ:}

Друк И.В., Снарская Д.И., Горощеня О.И. Потенциальная избыточность терапии сахарного диабета 2 типа в реальной клинической практике: данные регистра Омской области // Сахарный диабет. - 2021. — Т. 24. — №2. — С. $100-110$. doi: https://doi.org/10.14341/DM12469

\section{TO CITE THIS ARTICLE:}

Druk IV, Snarskaya DI, Goroshchenya OI. Potential overtreatment of type 2 diabetes therapy in real clinical practice: Omsk Oblast register data. Diabetes Mellitus. 2021;24(2):100-110. doi: https://doi.org/10.14341/DM12469 